Methods Consecutive patients attending a tertiary referral centre for clinically indicted endoscopic examination were prospectively recruited between August and December 2011. Sex, age, body mass index (BMI) and previous endoscopy experience were recorded. Procedural completion time, quality of bowel preparation and endoscopic findings were also documented. Patients were asked to grade anticipated and actual procedural discomfort and distress scores using a previously validated Numeric Rating Scale ranging form 0-10 as well as being asked to complete a Hospital Anxiety and Depression Scale. Patients also provided qualitative data, providing insights into their perceptions on perceived distress or discomfort. Data were analysed using SPSS version 19 with T-test analysis undertaken.

Results 271 patients were prospectively recruited (127 male, 144 female; median 56 years, range 17-89 years). Of these, 124 patients had a gastroscopy, 116 underwent colonoscopy and 31 had flexible sigmoidoscopy examinations. 34 patients (12.5\%) underwent bidirectional endoscopy. Analysis showed that discomfort scores were significantly higher in patients undergoing colonoscopy compared to gastroscopy (4.65 vs 2.90, $\mathrm{p}<0.001)$ and also when comparing flexible sigmoidoscopy to gastroscopy (4.10 vs $2.90, p=0.047)$. No difference was identified when comparing flexible sigmoidoscopy discomfort levels to colonoscopy $(\mathrm{p}=0.365)$. Interestingly, while discomfort scores were significantly lower in the gastroscopy group, overall distress levels were significantly higher in this group compared to the colonoscopy group (3.99 vs 3.16, $\mathrm{p}=0.049$ ). Data provided from the qualitative analysis would suggest that this is primarily due to the distress caused by oesophageal intubation.

Conclusion This is the first study to discriminate between distress and discomfort in endoscopic procedures and highlights variations in tolerability dependent on the underlying procedure undertaken. Our observations provides evidence to suggest greater attention should be made by endoscopists during oesophageal intubation during gastroscopy and with regards to gas insufflation during lower gastrointestinal endoscopic examinations.

Competing interests None declared.

\section{OC-146 OUTCOME OF ENDOSCOPIC SUBMUCOSAL DISSECTION (ESD) FOR SUPERFICIAL OESOPHAGEAL NEOPLASM: A UK PILOT SERIES}

doi:10.1136/gutjnl-2012-302514a.146

${ }^{1} \mathrm{~T}$ P George, ${ }^{*} \mathrm{R}$ Shakespeare, ${ }^{1} \mathrm{M}$ Collins, ${ }^{2} \mathrm{~A}$ Burdge. ${ }^{1}$ Department of Gastroenterology, Cross Border Upper GI Cancer Centre, Maelor Hospital, Wrexham, UK; ${ }^{2}$ Department of Histopathology, Cross Border Upper GI Cancer Centre, Maelor Hospital, Wrexham, UK

Introduction In Japan endoscopic submucosal dissection (ESD) is accepted as a safe and effective treatment for early oesophageal cancer. Experience in the UK remains limited and oesophagectomy is still the gold standard. The aim of this prospective single centre pilot study was to evaluate the safety and clinical outcomes of oesophageal ESD in a UK setting.

Methods Between July 2008 and November 2011 the regional upper GI MDT for North Wales and Cheshire considered 14 patients with early oesophageal cancer (T1N0M0) $(\mathrm{n}=11)$ and high grade dysplasia $(n=3)$ for ESD after full staging. All patients underwent trimodal endoscopy (autofluorescence, narrow band imaging, magnification, and chromoendoscopy) to assess the lesion and depth of invasion. Informed consent was obtained after full discussion and counselling as to alternative treatment options. Standard ESD technique was used, whereby the lesion was isolated by circumferential cutting using a flush and IT2 knife after marking the edges and raising with submucosal injection; followed by dissection. Specimens were staged according to the Kikuchi classification.
Patients with residual Barrett's $(\mathrm{n}=5$ ) had radio-frequency ablation after ESD to reduce the risk of metachronous cancer. Data were collected prospectively and audited by an independent group.

Results Of the 14 cases (nine male, five female; mean age 73 years), two were excluded as trimodal endoscopy showed evidence of deep submucosal infiltration and one patient declined treatment. Mean specimen size was $16 \mathrm{~mm}$. Procedure time ranged from 120 to $210 \mathrm{~min}$. Enbloc resection rate was $91 \%$. R0 resection rate of the lateral and deep margins were $82 \%$ and $64 \%$ respectively (Abstract OC-146 table 1). There were no major complications, although one procedure was abandoned as the endoscopic field of view was obscured by bleeding. Mean hospital stay was $72 \mathrm{~h}$. Procedure and disease specific mortality was zero. Over a median follow-up period of 20.5 months there was one recurrence. This occurred in a patient with incomplete resection of both lateral and deep margins at ESD. Those with R1 resection of the deep margins showed no evidence of recurrence.

Abstract OC-146 Table 1 Complete resection and complication rates of endoscopic submucosal dissection

\begin{tabular}{ll}
\hline & $\mathbf{n}(\%)$ \\
\hline ESD & 11 \\
Enbloc resection rate & $10(91)$ \\
R0 lateral margin & $9(82)$ \\
R0 deep margin & $7(64)$ \\
Major complications & 0 \\
Minor complication (minor bleeding) & $1(9)$ \\
Disease specific mortality & 0 \\
Recurrence & $1(9)$ \\
\hline
\end{tabular}

Conclusion ESD is a safe and effective treatment with high cure rate for early oesophageal neoplasm, even when the endoscopist is in the steep part of the learning curve. ESD has the advantage of high enbloc resection rates and low risk of recurrence. In our opinion all patients in the UK with early oesophageal cancer and high grade dysplasia should have access to ESD as a standard treatment option.

Competing interests None declared.

\section{OC-147 MULTICENTRE (CERT-N) AUDIT OF EXPERIENCE AND OUTCOMES OF ENDOSCOPIC BALLOON DILATATION TO TREAT CROHN'S DISEASE STRICTURES}

doi:10.1136/gutjnl-2012-302514a.147

${ }^{1} \mathrm{M}$ Bhalme, ${ }^{*}{ }^{2} \mathrm{E}$ Hargreaves, ${ }^{3} \mathrm{~T}$ Gledhill, ${ }^{2} \mathrm{Y}$ Prasad, ${ }^{2} \mathrm{~J}$ Geraghty, ${ }^{2} \mathrm{~S}$ Sarkar, ${ }^{4} \mathrm{R}$ Willert ${ }^{1}$ North Manchester General Hospital, Manchester, UK; ${ }^{2}$ Royal Liverpool and Broadgreen University Hospitals, Liverpool, UK; ${ }^{3}$ Aintree University Hospital, Aintree, UK; ${ }^{4}$ Central Manchester University Hospitals, Manchester, UK

Introduction Strictures are a common complication of Crohn's disease (CD), both de novo and following surgery (Sx). While endoscopic balloon dilatation (EBD) offers a valuable alternative to $\mathrm{Sx}$ in managing them, there is paucity of data on factors that may influence the safety and efficacy of this technique. Our aim was to perform a multi-centre audit to determine our experience and outcomes of EBD in symptomatic CD strictures.

Methods A retrospective audit across three major hospitals in Northwest England was performed on patients between 1998 and 2011. Demographics, smoking status, immunomodulation, CRP, endoscopic findings, EBD details including complications and subsequent surgery at follow-up were all recorded. Success of EBD was defined as symptomatic improvement without need for surgery at follow-up.

Results Patient \& Disease Demographics: 71 patients (43 female; age range $17-85$ years, median 47 ) were audited. Duration of CD was 